\title{
Article \\ Reservoir computing with delayed input for fast and easy optimization
}

\author{
Lina Jaurigue $^{1 *}$, Elizabeth Robertson ${ }^{2,}$, Janik Wolters ${ }^{2,}$, and Kathy Lüdge ${ }^{3,}$ \\ 1 Technische Universität Berlin; Institute of Theoretical Physics; linajaurigue@tu-berlin.de \\ 2 Technische Universität Berlin; Institut für Optik und Atomare Physik, janik.wolters@tu-berlin.de \\ 3 Technische Universität Ilmenau; Institute of Physics; kathy.luedge@tu-ilmenau.de \\ * Correspondence: linajaurigue@tu-berlin.de
}

\begin{abstract}
Reservoir computing is a machine learning method that uses the response of a dynamical system to a certain input in order to solve a task. As the training scheme only involves optimising the weights of the responses of the dynamical system, this method is particularly suited for hardware implementation. Furthermore, the inherent memory of dynamical systems which are suitable for use as reservoirs mean that this method has the potential to perform well on time series prediction tasks, as well as other tasks with time dependence. However, reservoir computing still requires extensive task dependent parameter optimisation in order to achieve good performance. We demonstrate that by including a time-delayed version of the input for various time series prediction tasks, good performance can be achieved with an unoptimised reservoir. Furthermore, we show that by including the appropriate time-delayed input, one unaltered reservoir can perform well on six different time series prediction tasks at a very low computational expense. Our approach is of particular relevance to hardware implemented reservoirs, as one does not necessarily have access to pertinent optimisation parameters in physical systems but the inclusion of an additional input is generally possible.
\end{abstract}

Keywords: reservoir computing; time series prediction; performance optimisation

\section{Introduction}

Reservoir computing $(\mathrm{RC})$ is a machine learning method that is particularly suited to solving dynamical tasks [1]. It was introduced as a way of using recurrent networks for machine learning but circumventing the costly training of the network weights [2]. The main principle underpinning reservoir computing is that the reservoir projects the inputs into a sufficiently high dimensional phase space such that it suffices to linearly sample the response of the reservoir in order to approximate the desired target for a given task. For this to work the reservoir must fulfil certain criteria; the response to sufficiently different inputs must be linearly separable, the reservoir must be capable of performing nonlinear transforms, and the reservoir must have the fading memory property [2]. However, even when these criteria are fulfilled, the performance depends greatly on the dynamics of the reservoir. Hence, in the past two decades a lot of research in the reservoir computing community has focused on the optimisation of the reservoir parameters [3-9]. Furthermore, the optimisation of the reservoir is a task specific problem [1,10-12] and a universal reservoir, which performs well on a range of tasks, remains elusive.

In a recent paper [13], the authors aim to eliminate the issue of hyperparameter optimisation altogether by removing the reservoir. Their approach essentially takes the well known nonlinear vector autoregression (NVAR) method, uses a less parsimonious approach to filling the feature vector and adds Tikhonov regularisation. However, the method of [13] trades the optimisation of the reservoir hyperparameters for the optimisation of the feature vector elements and it can not be asserted that the latter is generally less costly. Furthermore, one of the main factors driving research into reservoir 
computing forward is the possibility for hardware implementation [14-19], which is impractical when the reservoir is absent.

In this contribution we demonstrate a new approach that reduces the need for hyperparameter optimisation and is well suited to boosting the performance of physically implemented reservoir computers. Specifically, we show that, by adding a time delayed version of the input for a given task, the performance of an unoptimised reservoir can be greatly improved. We demonstrate this by using one unaltered reservoir to perform six different time series prediction tasks. In each case the only optimisation parameters are the delay and input strength of the additional delayed input. The aim of this work is not to achieve the best possible performance, but rather to demonstrate that reasonable performance can be achieved for various tasks using the same reservoir and at a very low computational cost.

Using time delayed input is a common approach for adding memory to feedforward networks [20-23] and is the basis of statistical forecasting methods [21,24]. However, despite the simplicity of this idea, to the best of our knowledge time-delayed inputs have not been used to optimise the performance of reservoir computers. This may be because the focus has been on constructing reservoirs that have the necessary memory to perform a given task [1].

Our results are of particular relevance to the hardware implementation of reservoir computing, because in physical systems one does not always have access to the relevant hyperparameters necessary for optimisation of the task dependent performance but it should always be possible to add an additional input.

\section{Methods}

In the following we describe the reservoir computing concept, the model for the reservoir, our proposed time-delayed input method and the benchmarking tasks that are used to test our approach.

\subsection{Reservoir computing}

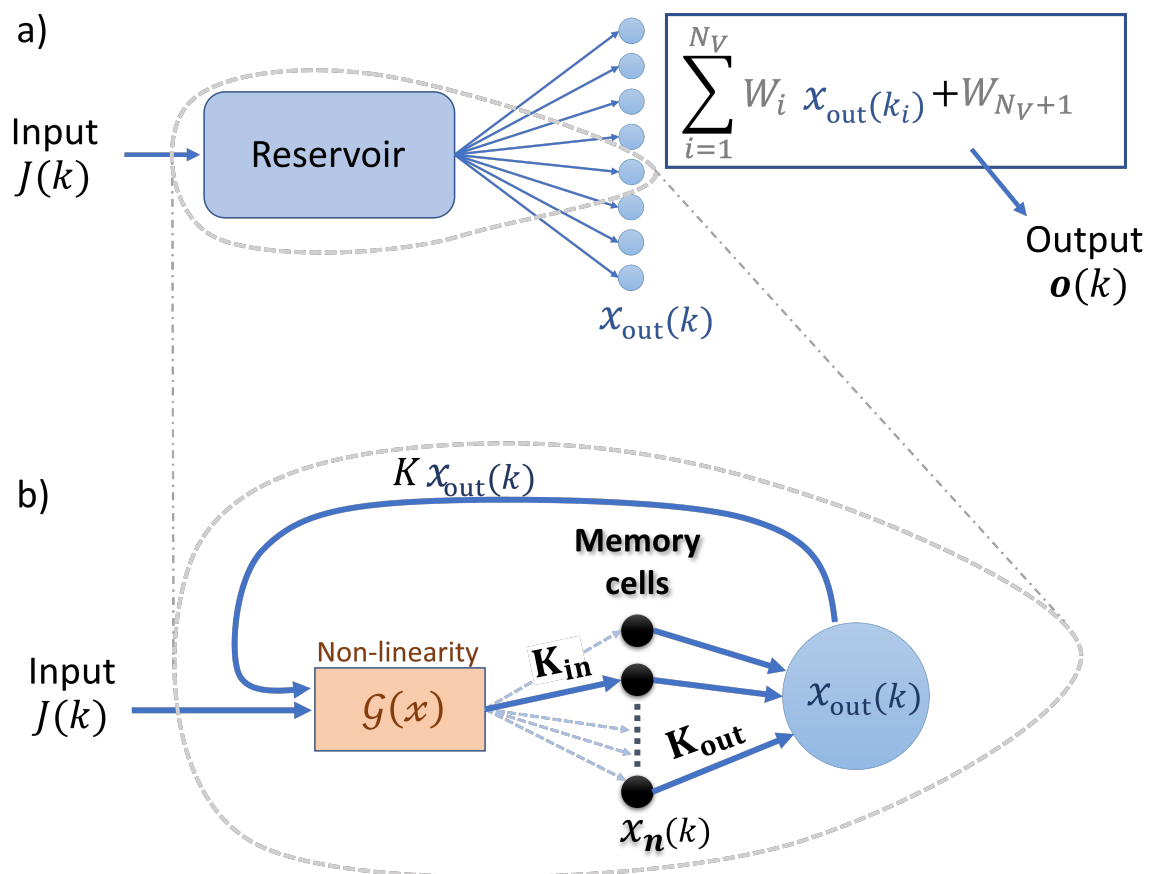

Figure 1. (a) Sketch of the reservoir computing concept. (b) Sketch of the memory cell reservoir described in Section 2.2. 
In reservoir computing the reservoir, which at this point can be treated as a black box, is fed an input and the response of the system is sampled a number of times. The responses are then linearly combined to approximate the desired output (see Fig.1 a). The linear output weights are trained via linear regression, typically using Tikhonov regularisation or regularisation by noise [1]. A variant of reservoir computing, that is of particular relevance for hardware implementation, is time-multiplexed reservoir computing using only one nonlinear element [25]. In this scheme both the injection of the data into the reservoir, and the filling of state matrix $\mathbf{S}$ occur sequentially. Typically a mask is applied to the input data in order to diversify the response of the reservoir to the input. In the training phase, the reservoir is fed a sequence of training data of length $K_{\mathrm{tr}}$. A mask of length $N_{v}$ is applied to each element of the training data, where $N_{v}$ corresponds to the readout dimension (i.e. the number virtual nodes). Hence, there are $N_{v} K_{\text {tr }}$ time-multiplexed inputs that are sequentially fed into the reservoir. The corresponding state matrix, which has the dimensions $K_{\operatorname{tr}} \times\left(N_{v}+1\right)$, is filled row by row with an additional bias term of 1 at the end of each row. The training step is then to find the $\left(N_{v}+1\right)$ dimensional weight vector $\mathbf{W}$ that best approximates

$$
\hat{\mathbf{o}} \approx \mathbf{S} \cdot \mathbf{W}
$$

where $\hat{\text { o }}$ is the vector of $K_{\mathrm{tr}}$ target outputs. The solution to this linear problem is given by

$$
\mathbf{W}=\left(\mathbf{S}^{\mathrm{T}} \mathbf{S}+\lambda \mathbf{I}\right)^{-\mathbf{1}} \mathbf{S}^{\mathrm{T}} \hat{\mathbf{o}}
$$

where $\lambda$ is the Tikhonov regularisation parameter and $\mathbf{I}$ is the identity matrix.

\subsubsection{Error measure}

To quantify the performance of the reservoir computer we use the normalised root mean squared error (NRMSE) defined as

$$
\mathrm{NRMSE}=\sqrt{\frac{\sum_{k=1}^{K_{o}}\left(\hat{o}_{k}-o_{k}\right)^{2}}{K_{o} \operatorname{var}(\hat{\mathbf{o}})}},
$$

where $\hat{o}_{k}$ are the target values, $o_{k}$ are the outputs produced by the reservoir computer and $K_{o}$ is the length of the vector ô.

\subsection{Reservoir Model}

To investigate the effect of delayed input on a physically implemented reservoir computer, we model a physical system that is inspired by optical delay line reservoirs $[26,27]$. Delay line implementations have shown promise due to high throughput speeds [28]. However, complex network connectivity, achieved via the introduction of multiple delays, represents a significant experimental hurdle, or requires opto-electrical conversion of the signal for electronic storage thereby forgoing the advantages of an alloptical implementation. Recent developments in optical quantum memories with high bandwidth [29] and high capacity [30] allow for the on-demand storage and retrieval of optical pulses and thus the implementation of delays of arbitrary length, limited only by the coherence time of the optical memory, which can reach up to one second [31]. The reservoir model described below models a physical optical system including the optical memory for the reconfigurable and arbitrary coupling of the injected information (modeled as memory cells with input and output coupling), a non-linear element (modeled as a semiconductor optical amplifier), and a short delay line; whose purpose is albeit not for introducing delay, but to recouple existing information back into the optical system. A sketch of the envisaged setup is shown in Fig. 1 b. Input is fed through a nonlinear element and then stored in memory cells. Combinations of the memory cells are then partially read out and coupled back into the nonlinear element. The map describing this 
process is given by the following. Let $x_{n}(k)$ be the state of the $n^{\text {th }}$ memory cell at time step $k$. The next time step is then given by

$$
\begin{gathered}
x_{n}(k+1)=\mathbf{K}_{\mathbf{i n} n}^{m} \mathcal{G}\left(K x_{\text {out }}(k)+J(k+1)\right)+\mathbf{K}_{\mathbf{n i n} n}^{m}\left(1-\mathbf{K}_{\text {out }_{n}}^{m}\right) x_{n}(k), \\
m \rightarrow(m+1) \bmod M
\end{gathered}
$$

where

$$
x_{\text {out }}(k)=\sum_{n=1}^{N} \mathbf{K}_{\text {out }}{ }_{n}^{m} x_{n}(k),
$$

$\mathcal{G}(x)$ is the function describing the nonlinear element, the matrices $\mathbf{K}_{\mathbf{i n}}, \mathbf{K}_{\mathbf{n i n}}$ and $\mathbf{K}_{\mathbf{o u t}}$ describe the coupling into and out of the memory cells, $K$ is the percentage of the output $x_{\text {out }}$ that is coupled back into the nonlinear element and $J(k)$ is the input. The coupling matrices have the dimensions $M x N$, where $N$ is the number of memory cells and $M$ is the period with which the coupling sequence repeats. For each iteration one row of the coupling matrices determines which memory cells are written into and which are read out of. $\mathbf{K}_{\mathbf{i n}}$ gives the write sequence and $\mathbf{K}_{\mathbf{o u t}}$ the out-coupling sequence. These two matrices contain values from zero to one. For $\mathbf{K}_{\text {in }}$ the row sum must be one. The entries of the matrix $\mathbf{K}_{\text {nin }}$ are

$$
\mathbf{K}_{\mathbf{n i n} n}{ }_{n}^{m}=\left\{\begin{array}{l}
0 \text { if } \mathbf{K}_{\mathbf{i n} n}^{m} \neq 0 \\
1 \text { if } \mathbf{K}_{\mathbf{i n} n}^{m}=0
\end{array} .\right.
$$

This allows the memory cells with new input to be overwritten and those without to be updated according to how much was read out.

The model described above allows for arbitrary coupling between the memory cells. For this study we choose $\mathbf{K}_{\mathbf{i n}}=\mathbf{K}_{\text {out }}=\mathbf{I}$, i.e. $M=N$. For this choice of coupling Eqs.(4) and (5) can be rewritten as

$$
x_{\text {out }}(k+1)=\mathcal{G}\left(K x_{\text {out }}(k-N+1)+J(k+1)\right) .
$$

We then choose $N=N_{v}+1$ where $N_{v}$ is the number of virtual nodes that will be used for the reservoir computing tasks. This coupling describes a type of ring coupling akin to delay-based reservoir computers with the feedback delay equal to $T+\theta$, where $T$ is the clock time and $\theta$ is the virtual node separation [27,32]. We choose such a simple coupling scheme as it has been demonstrated that such coupling topologies perform similarly to random coupling topologies [4]. Using Eq. (6) the rows of the state matrix $\mathbf{S}$ are filled with $N_{v}$ sequential $x_{\text {out }}(k)$ (Fig. 1).

For the nonlinearity we choose

$$
\mathcal{G}(x)=\frac{g_{0} x}{1+x / P_{\text {sat }}}
$$

which describes the input response of a semiconductor optical amplifier $[33,34]$.

\subsection{Input and mask}

The reservoir input is given by a task dependent time series and a time-delayed version of this time series. Before the data is fed into the reservoir, masks are applied to both input series. The masks consists of $N_{v}$ values drawn from a uniform distribution between 0 and 1 . The final input is then given by

$$
J(k)=G_{1} I\left(k^{\prime}\right) M_{1}\left(k^{\prime \prime}\right)+G_{2} I\left(k^{\prime}-d\right) M_{2}\left(k^{\prime \prime}\right)+J_{0},
$$

where $G_{1}$ and $G_{2}$ are the input scaling factors, $I\left(k^{\prime}\right)$ is the input time series, $d$ is the input delay, $M_{1}\left(k^{\prime \prime}\right)$ and $M_{2}\left(k^{\prime \prime}\right)$ are $k^{\prime \prime}$ th entries of the $N_{v}$ dimensional masking vectors and $J_{0}$ is a constant offset. A sketch of the masked input sequence is shown in Fig. 2. 


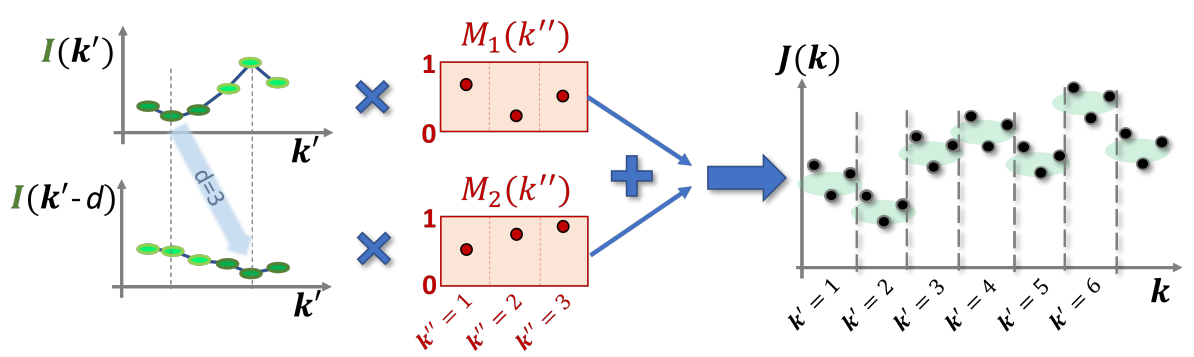

Figure 2. Sketch of the generation of the final input sequence $J(k)$ using the task dependent input $I\left(k^{\prime}\right)$, a delayed version of this input $I\left(k^{\prime}-d\right)$ and the masks $M_{1}\left(k^{\prime \prime}\right)$ and $M_{2}\left(k^{\prime \prime}\right)$, as described by Eq. (8).

\subsection{Time series prediction tasks}

\subsubsection{Mackey-Glass}

The Mackey-Glass equation is a delay differential equation which exhibits chaotic dynamics. The reservoir computing benchmarking task is to predict the timeseries $s$ number of steps ahead in the chaotic regime. The Mackey-Glass equation is [35]:

$$
\frac{d x}{d t}=\beta \frac{x(t-\tau)}{1+x(t-\tau)^{n}}-\gamma x
$$

We use the standard parameters: $\tau=17, n=10, \beta=0.2$ and $\gamma=0.1$. To create the input sequence $I\left(k^{\prime}\right)$ the time series generated by Eq. (9) is sampled with a time step of $d t=1$. The corresponding target sequence is then given by $I\left(k^{\prime}+s\right)$.

\subsubsection{NARMA10}

NARMA10 is a commonly used benchmarking task that is defined by the iterative formula

$$
A_{n+1}=0.3 A_{n}+0.05 A_{n}\left(\sum_{i=0}^{9} A_{n-i}\right)+1.5 u_{n-9} u_{n}+0.1,
$$

where $u_{n}$ are identically and independently drawn random numbers from a uniform distribution in the interval [0,0.5] [36]. The reservoir input sequence $I\left(k^{\prime}\right)$ is given by the sequence of $u_{n}$ and the target sequence is given by the corresponding $A_{n}$.

\subsubsection{Lorenz}

The Lorenz system [37] is given by

$$
\frac{d x}{d t}=c_{1} y-c_{1} x, \quad \frac{d y}{d t}=x\left(c_{2}-z\right)-y, \quad \text { and } \quad \frac{d z}{d t}=x y-c_{3} z .
$$

With $c_{1}=10, c_{2}=28$ and $c_{3}=8 / 3$ this system exhibits chaotic dynamics. We use the $x$ variable, sampled with a step size of $d t=0.02$, as the input $I\left(k^{\prime}\right)$ for two time series prediction tasks. The first is one step ahead $(s=1)$ prediction of the $x$ variable. The second is one step ahead $(s=1)$ cross prediction of the $z$ variable. 


\subsection{Simulation conditions}

Table 1. Reservoir and input parameter values.

\begin{tabular}{cccc}
\hline Parameter & Value & Parameter & Value \\
\hline$g_{0}$ & 40 & $P_{\text {sat }}$ & 1 \\
$K$ & 0.02 & $N$ & 31 \\
$N_{v}$ & 30 & $\lambda$ & $5 \mathrm{e}-6$ \\
$G_{1}$ (Mackey-Glass) & 1 & $J_{0}$ (Mackey-Glass) & 0 \\
$G_{1}$ (Lorenz) & 0.03 & $J_{0}$ (Lorenz) & 0.85 \\
$G_{1}$ (NARMA10) & 1.8 & $J_{0}$ (NARMA10) & 0.4 \\
\hline
\end{tabular}

For all tasks the reservoir is initialised with an input sequence $I\left(k^{\prime}\right)$ of length 10000 . The system is then trained on $K_{\mathrm{tr}}=10000$ inputs. This is followed by another buffer of 10000 inputs, before the performance is tested on a sequence of $K_{\mathrm{te}}=5000$ inputs, unless stated otherwise. For each task the reservoir parameters are kept identical and are as given in Table 1. The input scaling of the primary input $G_{1}$ (non-delayed input) and the offset $J_{0}$ are scaled such that the input range for each task is approximately [0.4,1.3]. The scaling of the delayed input $G_{2}$ and the input delay $d$ are used as the optimisation parameters. For each task the performance is averaged over 100 realisations of the random masks and in the case of NARMA10 also the random inputs.

\section{Results}

The performance of the reservoir with additional delayed input is tested on six tasks; we first consider Mackey-Glass time series prediction for one, three and ten steps into the future. The results of the Mackey-Glass tasks, and their relation to the delayed input parameters, are depicted in Fig.3 a-c. Corresponding scans of the delayed input strength $G_{2}$ for the optimal input delay $d$, for each task, are shown in Fig. 4 a-c. $G_{2}=0$ corresponds to the system without delayed input and should be used as the reference to quantify the performance boost due to the delayed input. For each of the three cases the delayed input leads to a reduction in the NRMSE, ranging from $20 \%$ for $s=1$ to over a factor three for $s=10$. The optimal values for the delay and the input scaling $G_{2}$ vary depending on the number of steps $s$ predicted into the future. In agreement with the results presented in [38], larger input scaling is required as $s$ increases, indicating that nonlinear transforms become increasingly important. In terms of the absolute performance, similar results are achieved compared with other studies [38,39], despite the number of virtual nodes used in this study being significantly lower.

The results for the NARMA10 task are shown in Fig. $3 \mathrm{~d}$ and Fig. $4 \mathrm{~d}$. Without the delayed input $\left(G_{2}=0\right)$ the performance of the reservoir is very poor. This is in contrast to the Mackey-Glass $s=1$ for which the performance without delayed input (Fig. 3a with $G_{2}=0$ ) is reasonable. Moreover, this finding supports the general observation that reservoir computers have to be optimised to individual tasks and perform poorly as universal approximators $[1,10,11]$. The inclusion of delayed input significantly reduced the NARMA10 error, reaching a NRMSE of about 0.3 for the input delay $d=9$. In absolute terms a NRMSE of 0.3 is within the range of typically quoted best values (NRMSE $=0.15-0.4)[4,10,40-43]$, however usually achieved with a much higher output dimension than the $N_{v}=30$ used here. The performance achieved in this study came at a very low computational cost. As a comparison, in [43] the authors investigate the influence of combining echo state networks with different timescales and achieve a best performance of just under 0.4 for the NRMSE, at a greater computational expense.

The remaining two tasks are one step ahead Lorenz $x$ prediction and one step head Lorenz $z$ cross prediction, the results of which are shown in Fig. 3e-f and Fig. 4e-f. In both cases there is an improvement in the performance with the correct choice of the delayed input. It is has been demonstrated that the Lorenz $x$ one step ahead prediction requires only the very recent history of the $x$-variable time series [13], and we find the optimal 

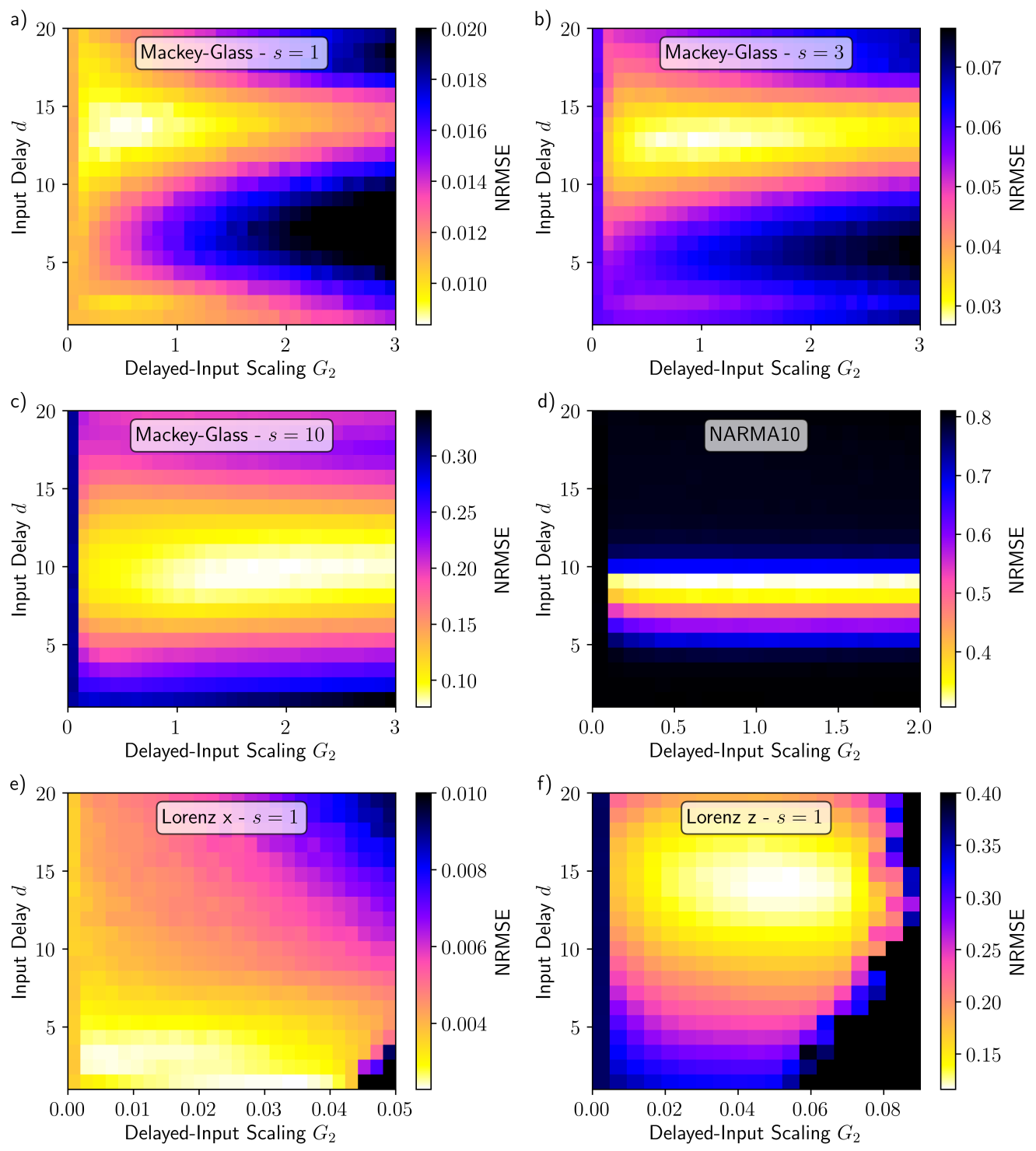

Figure 3. NRMSE as a function of the delayed input parameters $d$ and $G_{2}$ for Mackey-Glass (a) one, (b) three and (c) ten step ahead prediction, (d) NARMA10, (c) Lorenz $x$ one step ahead prediction and (f) Lorenz $z$ one step ahead cross prediction. Parameters are as stated in Sec. 2.5, except for (a) and (e) where $K_{\text {te }}=30000$.

input delay of $d=1$ to be consistent with this prior knowledge. For the Lorenz $z$ cross prediction task, on the other hand, there is a strong dependence on the history of the Lorenz $x$ variable. In this case the best performance is achieved when the second input is delayed by $d=14$ time steps. The optimal delayed-input scaling $G_{2}$ is larger for the Lorenz $z$ task than the Lorenz $x$ task (as seen by comparing the positions of the minima in Fig. 4 e and f), indicating that the cross prediction task requires a greater degree of nonlinearity as well as a longer memory.

In order to demonstrate that the improvement in the performance with delayed input is not specific to the reservoir parameters used for Fig. 3, in Fig. 5 we show the NRMSE for the Mackey-Glass $s=10$ task as a function of (a) the virtual node coupling strength $K$ and (b) the coupling delay $N$ (i.e. the number of memory cells). These parameters have a strong influence on the properties of the reservoir. In both cases the NRMSE without delayed input (orange dotted line) shows a large variation over the respective parameter ranges and is always larger than the error with optimised delayed input (blue dashed line). With optimised delayed input the variation in the error is 

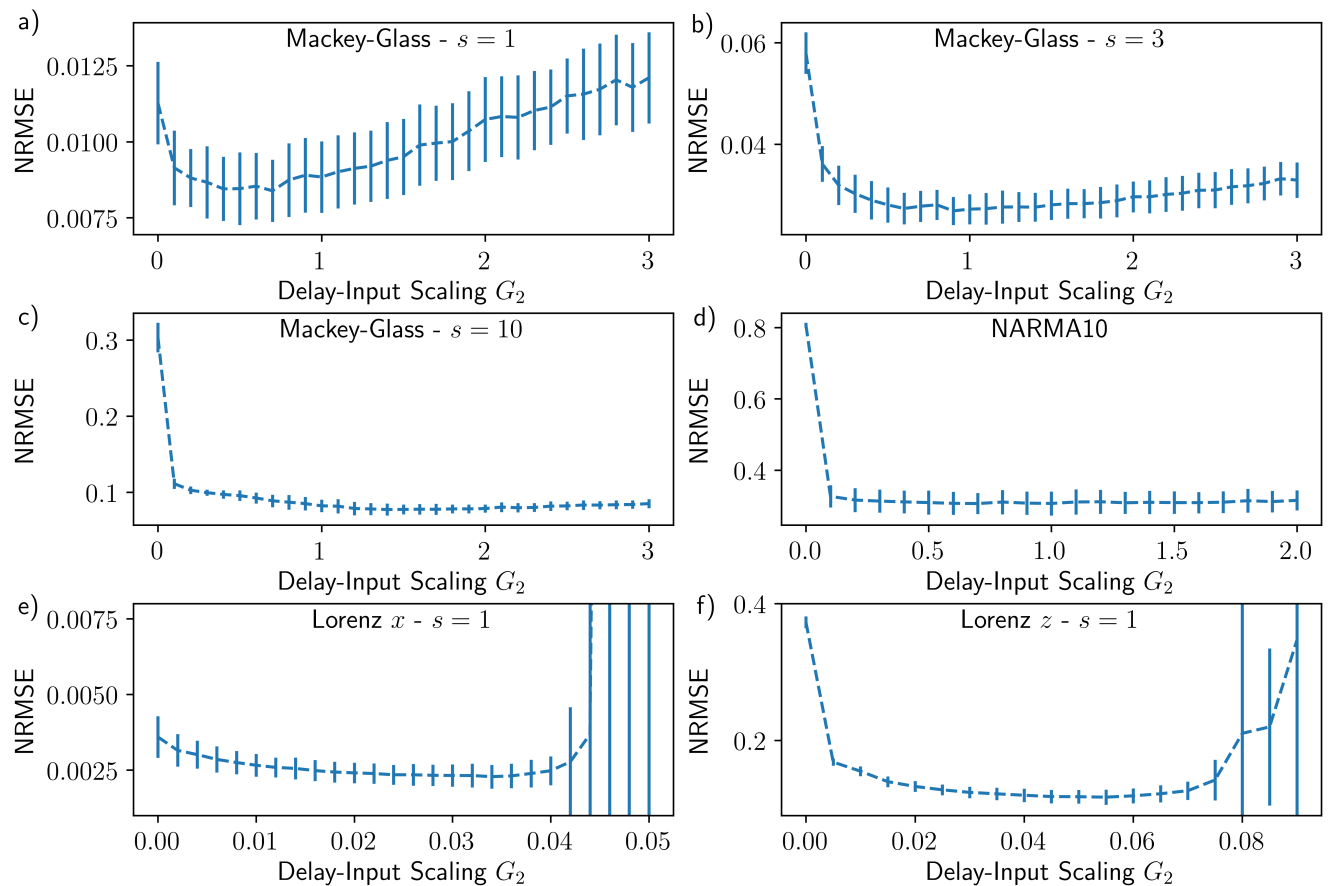

Figure 4. NRMSE for optimised input delay $d$, as a function of the delayed-input scaling $G_{2}$ for Mackey-Glass (a) one, (b) three and (c) ten step ahead prediction, (d) NARMA10, (c) Lorenz $x$ one step ahead prediction and (f) Lorenz $z$ one step ahead cross prediction. The error bars indicate the standard deviation. The optimal input delays are (a) $d=14,(\mathrm{~b}) d=13$, (c) $d=9$, (d) $d=9$, (e) $d=1$ and (f) $d=15$. The remaining parameters are as stated in Sec. 2.5, except for (a) and (e) where $K_{\mathrm{te}}=30000$.

comparatively small, demonstrating that the inclusion of the delayed input works well independent of the reservoir properties. The peak in the NRMSE at $N=30$ in Fig. $5 \mathrm{~b}$ is a well known resonance effect that occurs at resonances between the number of virtual nodes and the coupling range $N$, equivalent to clock time and delay resonances in time continuous systems [44].

To further demonstrate the universality of this method, we show the NARMA10 error with delayed input for a time-continuous reservoir in Fig. 6. In this case the reservoir is given by the Stuart-Landau equation with time-delayed feedback (see Appendix B). The reservoir parameters have not been optimised for the NARMA10 task, resulting in very poor performance without delayed input $\left(G_{2}=0\right)$. With optimised delayed input parameters reasonable performance is achieved, similar to the optimal results for the memory cell reservoir in Fig. 3 d. For the Stuart-Landau reservoir optimal performance is achieved for the input delay $d=10$. Whereas, for the memory cell reservoir the optimal input delay is $d=9$. This is because the required input delay depends both on the dynamics of the reservoir as well as the memory requirements of the particular task.

\section{Discussion}

We have shown that, for various time series prediction tasks, including a delayed version of the input can lead to a substantial improvement in the performance of a reservoir. We have demonstrated this using a simple map describing a semiconductor optical amplifier non-linearity and a ring-like coupling realized via memory cells. With this approach we were able to use one unaltered reservoir to perform well on six different tasks, each with different memory and nonlinear transform requirements. The performance boost due to the delayed input is achieved over a wide range of the reservoir parameters and was also demonstrated for a time-continuous system, indicating that our approach is applicable to a wide range of reservoirs. 

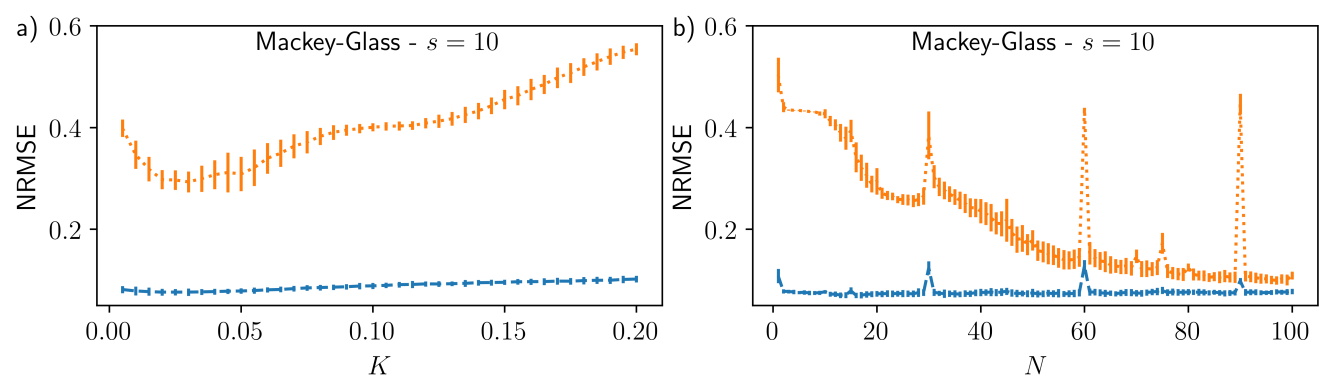

Figure 5. NRMSE for Mackey-Glass 10 step ahead prediction as a function of (a) the virtual node coupling strength $K$ and (b) the coupling delay $N$. The orange dotted (blue dashed) lines show the results without (with) delayed input. Along the blue curve the delayed input parameters $d$ and $G_{2}$ have been optimised (see Fig. A1 in Appendix A for their values). The error bars indicate the standard deviation. All remaining parameters are as stated in Sec. 2.5.

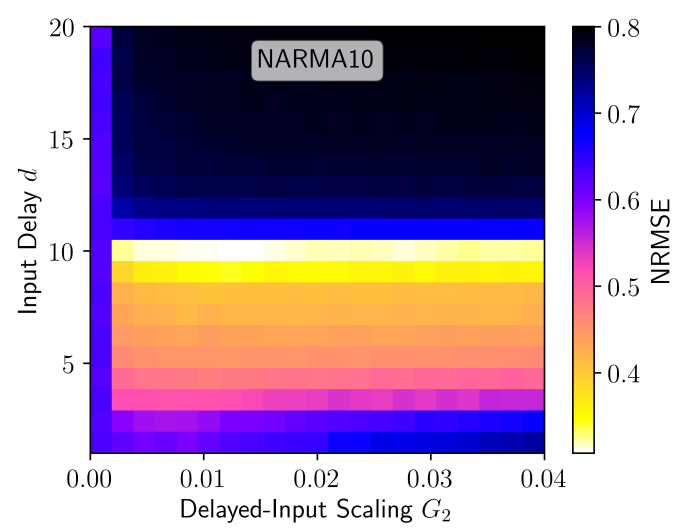

Figure 6. NRMSE for the NARMA10 task as a function of the delayed input parameters $d$ and $G_{2}$ using the Stuart-Landau delay-based reservoir computer described in Appendix B.

Our results are significant for a number of reasons. Firstly, we have demonstrated that computationally expensive hyperparameter optimisation can be circumvented by tuning only two input parameters. By including an additional delayed input, reasonable performance can be achieved using an unoptimised reservoir. Nevertheless, we note that depending on the requirements for a given task additional hyperparameter optimisation may be necessary. Secondly, to the best of our knowledge, this is the first demonstration of an identical reservoir performing well on such a large range of tasks. Thirdly, the simplicity of our approach means that it is well suited to be applied on physical reservoirs.

This study has raised several questions surrounding delay-based reservoir optimisation that require further investigation. For example, the optimal delayed-input parameters are task dependent and how these relate to a given task is not fully understood. The NARMA10 results presented in this study indicate that the optimal delayed-input parameters are related both to the reservoir and requirements of the task. This means that it may be possible to not only use reservoir computing for real world time series prediction tasks, but also to gain insights into the dynamical systems being investigated. For example, in tasks such as El Nino prediction where the underlying dynamical system is very complex and the relevant physical processes are not fully understood [45]. Here, investigations surrounding delay-based input could provide critical insight into the involved timescales. Furthermore, the minimum requirements for a reservoir to yield good performance on a range of tasks by only tuning the delayed input parameters, remain to be determined.

A natural extension of our proposed approach is to include multiple delayed input terms. This would bring the reservoir computing approach closer to classical statistical forecasting methods such as NVAR and could lead to a further improved 
performance, especially for tasks involving multiple disparate timescales. However, possible performance improvement with added input terms must be weighed against the associated increase in the computational cost as each added input adds two new optimisation parameters.

Author Contributions: Conceptualization, L.J., J.W. and K.L.; methodology, L.J.; software, L.J.; validation, L.J.; formal analysis, L.J.; investigation, L.J. and E.R.; writing-original draft preparation, L.J. ; writing—review and editing, L.J., E.R., J.W. and K.L.; visualization, L.J. and K.L.; funding acquisition, L.J., J.W. and K.L. All authors have read and agreed to the published version of the manuscript.

Funding: This research was funded by the Deutsche Forschungsgemeinschaft (DFG), grant number LU 1729/3-1. E.R. acknowledges funding through the Helmholtz Einstein International Berlin Research School in Data Science (HEIBRiDS).

Conflicts of Interest: The authors declare no conflict of interest.

\section{Abbreviations}

The following abbreviations are used in this manuscript:

RC Reservoir computing

NVAR Nonlinear vector autoregression

NRMSE Normalise root mean squared error

\section{Appendix A Optimised input parameters}
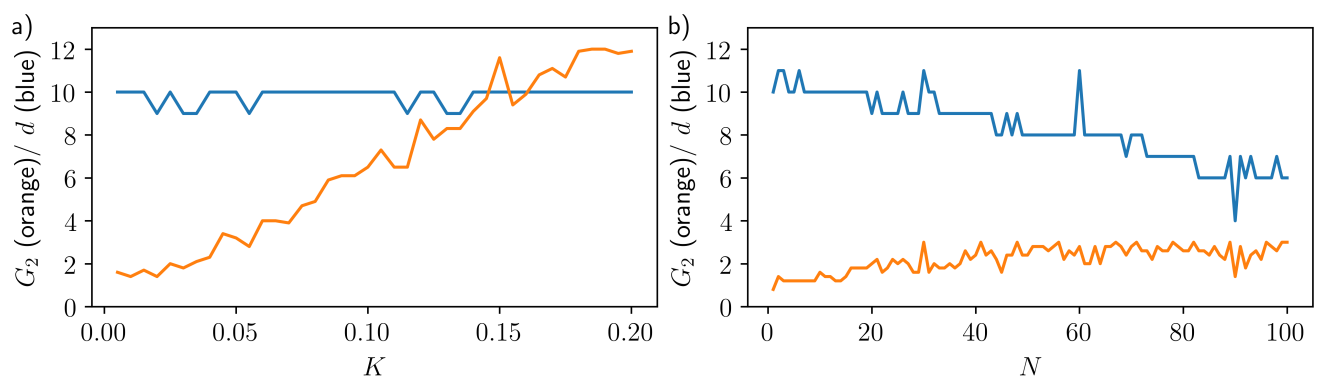

Figure A1. Values for the optimised input parameters $G_{2}$ (orange) and $d$ (blue), corresponding to the Mackey-Glass $s=10$ results depicted in Fig. 5, as a function of (a) the virtual node coupling strength $K$ and (b) the coupling delay $N$. All remaining parameters are as stated in Sec. 2.5.

The optimised values of the delayed-input scaling $d$ and the input delay $d$ corresponding to Fig. 5 are given in Fig. A1.

\section{Appendix B Stuart-Landau delay-based reservoir computer}

The Stuart-Landau system with time delayed feedback is given by

$$
\frac{d Z}{d t}=\left(\lambda_{S L}+J(t)+i \omega+\gamma|Z|^{2}\right) Z+K e^{i \phi} Z(t-\tau) .
$$

The parameter values are given in Table A1. The input sequence $J(t)$ for this system is comprised of piece-wise constant steps of length $\theta=T / N_{v}, T$ is the clock time [42]. For this system we use regularisation by noise, meaning that we added Gaussian white noise of strength $R_{\text {noise }}$ to the state matrix $\mathbf{S}$ entries. 
Table A1. Reservoir and input parameter values for the Stuart-Landau RC.

\begin{tabular}{cccc}
\hline Parameter & Value & Parameter & Value \\
\hline$\lambda_{S L}$ & -0.02 & $\omega$ & 0 \\
$\gamma$ & -0.1 & $K$ & 0.1 \\
$\tau$ & 105 & $\phi$ & 0 \\
$N_{v}$ & 30 & $R_{\text {noise }}$ & $1 \mathrm{e}-7$ \\
$G_{1}$ & 0.01 & $J_{0}$ & 0 \\
$T$ & 80 & & \\
\hline
\end{tabular}

\section{References}

1. Nakajima, K.; Fischer, I. Reservoir Computing: Theory, Physical Implementations, and Applications; 2021.

2. Jaeger, $\mathrm{H}$. The 'echo state' approach to analysing and training recurrent neural networks. GMD Report 148, GMD - German National Research Institute for Computer Science, 2001. doi:publica.fraunhofer.de/documents/b-73135.html.

3. Dutoit, X.; Schrauwen, B.; Van Campenhout, J.; Stroobandt, D.; Van Brussel, H.; Nuttin, M. Pruning and regularization in reservoir computing. Neurocomputing 2009, 72, 1534-1546. Advances in Machine Learning and Computational Intelligence, doi:https://doi.org/10.1016/j.neucom.2008.12.020.

4. Rodan, A.; Tiňo, P. Minimum Complexity Echo State Network. IEEE Trans. Neural Netw. 2011, 22. doi:10.1109/tnn.2010.2089641.

5. Grigoryeva, L.; Henriques, J.; Larger, L.; Ortega, J.P. Stochastic nonlinear time series forecasting using time-delay reservoir computers: Performance and universality. Neural Netw. 2014, 55, 59.

6. Nguimdo, R.M.; Verschaffelt, G.; Danckaert, J.; Van der Sande, G. Simultaneous Computation of Two Independent Tasks Using Reservoir Computing Based on a Single Photonic Nonlinear Node With Optical Feedback. IEEE Trans. Neural Netw. Learn. Syst. 2015, 26. doi:10.1109/tnnls.2015.2404346.

7. Griffith, A.; Pomerance, A.; Gauthier, D.J. Forecasting chaotic systems with very low connectivity reservoir computers. Chaos 2019, 29, 123108, [https:/ / doi.org/10.1063/1.5120710]. doi:10.1063/1.5120710.

8. Carroll, T.L. Path length statistics in reservoir computers. Chaos 2020, 30, 083130, [https://doi.org/10.1063/5.0014643]. doi:10.1063/5.0014643.

9. Zheng, T.Y.; Yang, W.H.; Sun, J.; Xiong, X.Y.; Li, Z.T.; Zou, X.D. Parameters optimization method for the time-delayed reservoir computing with a nonlinear duffing mechanical oscillator. Sci. Rep. 2021, 11, 997. doi:10.1038/s41598-020-80339-5.

10. Ortín, S.; Pesquera, L. Reservoir Computing with an Ensemble of Time-Delay Reservoirs. Cognitive Computation 2017, 9, 327-336. doi:10.1007/s12559-017-9463-7.

11. Röhm, A.; Lüdge, K. Multiplexed networks: reservoir computing with virtual and real nodes. J. Phys. Commun. $2018,2,085007$. doi:10.1088/2399-6528/aad56d.

12. Brunner, D. Photonic Reservoir Computing, Optical Recurrent Neural Networks; De Gruyter, 2019.

13. Gauthier, D.J.; Bollt, E.M.; Griffith, A.; Barbosa, W.A.S. Next generation reservoir computing. Nat. Commun. 2021, $12,5564$. doi:10.1038/s41467-021-25801-2.

14. Vandoorne, K.; Dambre, J.; Verstraeten, D.; Schrauwen, B.; Bienstman, P. Parallel reservoir computing using optical amplifiers. IEEE Trans. Neural Netw. 2011, 22, 1469-1481. doi:10.1109/tnn.2011.2161771.

15. Duport, F.; Schneider, B.; Smerieri, A.; Haelterman, M.; Massar, S. All-optical reservoir computing. Opt. Express 2012, 20, 22783-22795.

16. Tanaka, G.; Yamane, T.; Héroux, J.B.; Nakane, R.; Kanazawa, N.; Takeda, S.; Numata, H.; Nakano, D.; Hirose, A. Recent advances in physical reservoir computing: A review. Neural Netw. 2019, 115, 100-123. doi:https://doi.org/10.1016/j.neunet.2019.03.005.

17. Canaday, D.; Griffith, A.; Gauthier, D.J. Rapid time series prediction with a hardware-based reservoir computer. Chaos 2018, 28, 123119, [https://doi.org/10.1063/1.5048199]. doi:10.1063/1.5048199.

18. Harkhoe, K.; Verschaffelt, G.; Katumba, A.; Bienstman, P.; Van der Sande, G. Demonstrating delay-based reservoir computing using a compact photonic integrated chip. Opt. Express 2020, 28, 3086. doi:10.1364/oe.382556.

19. Freiberger, M.; Sackesyn, S.; Ma, C.; Katumba, A.; Bienstman, P.; Dambre, J. Improving Time Series Recognition and Prediction With Networks and Ensembles of Passive Photonic Reservoirs. IEEE J. Sel. Top. Quantum Electron. 2020, $26,7700611$.

20. Waibel, A.; Hanazawa, T.; Hinton, G.E.; Shikano, K.; Lang, K.J. Phoneme recognition using time-delay neural networks. IEEE Trans. Signal Process. 1989, 37, 328-339. doi:10.1109/29.21701.

21. Karamouz, M.; Razavi, S.; Araghinejad, S. Long-lead seasonal rainfall forecasting using time-delay recurrent neural networks: a case study. Hydrol. Process. 2008, 22, 229-241, [https://onlinelibrary.wiley.com/doi/pdf/10.1002/hyp.6571]. doi:https://doi.org/10.1002/hyp.6571.

22. Han, B.; Han, M. An Adaptive Algorithm of Universal Learning Network for Time Delay System. 2005 International Conference on Neural Networks and Brain, 2005, Vol. 3, pp. nil19-1744. doi:10.1109/icnnb.2005.1614964.

23. Ranzini, S.M.; Da Ros, F.; Bülow, H.; Zibar, D. Tunable Optoelectronic Chromatic Dispersion Compensation Based on Machine Learning for Short-Reach Transmission. Appl. Sci. 2019, 9. doi:10.3390/app9204332. 
24. Bardella, P.; Drzewietzki, L.; Krakowski, M.; Krestnikov, I.; Breuer, S. Mode locking in a tapered two-section quantum dot laser: design and experiment. Opt. Lett. 2018, 43, 2827-2830. doi:10.1364/ol.43.002827.

25. Appeltant, L.; Soriano, M.C.; Van der Sande, G.; Danckaert, J.; Massar, S.; Dambre, J.; Schrauwen, B.; Mirasso, C.R.; Fischer, I. Information processing using a single dynamical node as complex system. Nat. Commun. 2011, 2, 468. doi:10.1038/ncomms1476.

26. Paquot, Y.; Duport, F.; Smerieri, A.; Dambre, J.; Schrauwen, B.; Haelterman, M.; Massar, S. Optoelectronic Reservoir Computing. Sci. Rep. 2012, 2. doi:10.1038/srep00287.

27. Brunner, D.; Penkovsky, B.; Marquez, B.A.; Jacquot, M.; Fischer, I.; Larger, L. Tutorial: Photonic neural networks in delay systems. J. Appl. Phys. 2018, 124, 152004. doi:10.1063/1.5042342.

28. Brunner, D.; Soriano, M.C.; Mirasso, C.R.; Fischer, I. Parallel photonic information processing at gigabyte per second data rates using transient states. Nat. Commun. 2013, 4, 1364. doi:10.1038/ncomms2368.

29. Wolters, J.; Buser, G.; Horsley, A.; Béguin, L.; Jöckel, A.; Jahn, J.P.; Warburton, R.J.; Treutlein, P. Simple Atomic Quantum Memory Suitable for Semiconductor Quantum Dot Single Photons. Phys. Rev. Lett. 2017, 119, 060502. doi:10.1103/physrevlett.119.060502.

30. Jiang, N.; Pu, Y.F.; Chang, W.; Li, C.; Zhang, S.; Duan, L.M. Experimental realization of 105-qubit random access quantum memory. npj Quantum Information 2019, 5, 28. doi:10.1038/s41534-019-0144-0.

31. Katz, O.; Firstenberg, O. Light storage for one second in room-temperature alkali vapor. Nature Communications 2018, 9, 2074. doi:10.1038/s41467-018-04458-4.

32. Arecchi, F.T.; Giacomelli, G.; Lapucci, A.; Meucci, R. Two-dimensional representation of a delayed dynamical system. Phys. Rev. A 1992, 45, R4225.

33. Zajnulina, M.; Lingnau, B.; Lüdge, K. Four-wave Mixing in Quantum Dot Semiconductor Optical Amplifiers: A Detailed Analysis of the Nonlinear Effects. IEEE J. Sel. Top. Quantum Electron. 2017, 23, 3000112. doi:10.1109/jstqe.2017.2681803.

34. Lingnau, B.; Lüdge, K. Quantum-Dot Semiconductor Optical Amplifiers. In Handbook of Optoelectronic Device Modeling and Simulation; Piprek, J., Ed.; CRC Press: Boca Raton, FL, USA, 2017; Vol. 1, Series in Optics and Optoelectronics, chapter 23. doi:10.1201/9781315152301.

35. Mackey, M.C.; Glass, L. Oscillation and chaos in physiological control systems. Science 1977, 197, 287. doi:10.1126/science.267326.

36. Atiya, A.F.; Parlos, A.G. New results on recurrent network training: unifying the algorithms and accelerating convergence. IEEE Trans. Neural Netw. 2000, 11, 697-709.

37. Lorenz, E.N. Deterministic nonperiodic flow. J. Atmos. Sci. 1963, 20, 130.

38. Goldmann, M.; Mirasso, C.R.; Fischer, I.; Soriano, M.C. Exploiting transient dynamics of a time-multiplexed reservoir to boost the system performance. 2021 International Joint Conference on Neural Networks (IJCNN), 2021, pp. 1-8. doi:10.1109/ijcnn52387.2021.9534333.

39. Ortín, S.; Soriano, M.C.; Pesquera, L.; Brunner, D.; San-Martín, D.; Fischer, I.; Mirasso, C.R.; Gutierrez, J.M. A Unified Framework for Reservoir Computing and Extreme Learning Machines based on a Single Time-delayed Neuron. Sci. Rep. 2015, 5, 14945. doi:10.1038/srep14945.

40. Köster, F.; Yanchuk, S.; Lüdge, K. Insight into delay based reservoir computing via eigenvalue analysis. J. Phys. Photonics 2021, 3, 024011. arXiv:2009.07928, doi:10.1088/2515-7647/abf237.

41. Köster, F.; Ehlert, D.; Lüdge, K. Limitations of the recall capabilities in delay based reservoir computing systems. Cogn. Comput. 2020, 2020. Springer, ISSN 1866-9956, doi:10.1007/s12559-020-09733-5.

42. Röhm, A.; Jaurigue, L.C.; Lüdge, K. Reservoir Computing Using Laser Networks. IEEE J. Sel. Top. Quantum Electron. 2019, 26, 7700108. doi:10.1109/jstqe.2019.2927578.

43. Manneschi, L.; Ellis, M.O.A.; Gigante, G.; Lin, A.C.; Del Giudice, P.; Vasilaki, E. Exploiting Multiple Timescales in Hierarchical Echo State Networks. Front. Appl. Math. Stat. 2021, 6, 76. doi:10.3389/fams.2020.616658.

44. Stelzer, F.; Röhm, A.; Lüdge, K.; Yanchuk, S. Performance boost of time-delay reservoir computing by non-resonant clock cycle. Neural Netw. 2020, 124, 158-169. doi:https:/ / doi.org/10.1016/j.neunet.2020.01.010.

45. Nooteboom, P.D.; Feng, Q.Y.; López, C.; Hernández-García, E.; Dijkstra, H.A. Using network theory and machine learning to predict El Niño. Earth System Dynamics 2018, 9, 969-983. doi:10.5194/esd-9-969-2018. 